\title{
Effect of multi-level stroke education on treatment and prognosis of acute ischemic stroke
}

\author{
XIAOMAN ZHANG ${ }^{*}$, YINFANG LIU* ${ }^{*}$ XINHUI CAO, XIAOYU XU, YATAO ZHU and CHAOGANG WANG \\ Department of Neurology, The First People's Hospital of Zhengzhou, Zhengzhou, Henan 450004, P.R. China
}

Received December 17, 2019; Accepted May 27, 2020

DOI: $10.3892 /$ etm.2020.9028

\begin{abstract}
This observational study aimed at the significance of multi-level education in the treatment and prognosis of acute ischemic stroke. Multi-level stroke education was carried out among residents and medical staff for one year in Guancheng district. After 1 year, 519 patients with acute ischemic stroke admitted to The First People's Hospital of Zhengzhou were invited to the study, 272 patients from the Guancheng district were divided into the experimental group, and 247 patients who were not from the Guancheng district but in the neighborhood of The First People's Hospital of Zhengzhou were divided into the control group. Statistical methods were applied to analyze the degree of awareness of stroke, the time from onset to hospital, the route to hospital, the number of patients coming to the hospital within $4.5 \mathrm{~h}$, the number of intravenous thrombolysis, door-to-needle time (DNT), modified Rankin scale (MRS) score, and the number of hemorrhagic transformation cases. After one year of multi-level systematic stroke education, there were significant differences in stroke awareness between the experimental group and the control group in terms of limb weakness (87.87 vs. $62.75 \%$ ), speech inarticulation (78.3 vs. $55.06 \%$ ), facial paralysis $(69.12$ vs. $38.06 \%)$, limb numbness (57.35 vs. $29.15 \%$ ), consciousness disorder (62.50 vs. $42.11 \%$ ), walking instability with severe dizziness (39.97 vs. $15.79 \%$ ) $(\mathrm{P}<0.05)$. There was no statistical significant difference in unclear vision or blind eyes or severe headache $(\mathrm{P}>0.05)$. There were statistical differences between the two groups in the time from the onset to the hospital (14.82 \pm 17.67 vs. $25.92 \pm 25.23)$, emergency medical services (EMS) (36.02 vs. 16.19\%), number of patients coming to the hospital within $4.5 \mathrm{~h}$ (67 vs. 32), venous thrombolysis cases (55 vs. 17), DNT time $(42.43 \pm 17.30$ vs. $63.35 \pm 26.53)$, hemorrhagic transformation cases (11 vs. 21 ), and MRS score grade $\geq 2$ (230 vs. 169)
\end{abstract}

Correspondence to: Dr Xiaoman Zhang, Department of Neurology, The First People's Hospital of Zhengzhou, 56 Dongdajie Avenue, Zhengzhou, Henan 450004, P.R. China

E-mail: md703o@163.com; zhangxiaoman2@126.com

*Contributed equally

Key words: stroke education, acute ischemic stroke, prognosis
$(\mathrm{P}<0.05)$. Multi-level education can effectively improve the patient's awareness of stroke, encourage more patients to use EMS system to the hospital. More patients were aware that they should reach the hospital within $4.5 \mathrm{~h}$. It helps shorten DNT time and give more patients the opportunity to receive intravenous thrombolysis or intravascular thrombectomy, which may improve the prognosis and reduce hemorrhagic transformation without reducing mortality.

\section{Introduction}

Acute ischemic stroke is a serious worldwide public health problem. It is the main cause of death and disability, ranking second among the causes of death among Chinese residents $(1,2)$. Vascular patency and cerebral tissue perfusion in acute phase are effective methods to reduce disability rate and mortality. Intravenous thrombolysis and intravascular mechanical thrombus are highly recommended as the treatment of vascular opening in the guidelines for the treatment of ischemic stroke in China and the guidelines for early management of acute ischemic stroke issued by the American Heart Association/American Stroke Association (AHA/ASA) $(3,4)$. By continuous research around the world, mechanical thrombus extraction in part of anterior circulation can be extended to $24 \mathrm{~h}$ by screening time window and $9 \mathrm{~h}$ by intravenous thrombolysis time window. But the two treatments are still highly timedependent. Therefore, it is very important to transfer stroke patients to eligible hospitals as soon as possible $(5,6)$. The chance of intravenous thrombolysis in China is low, only $2.4 \%$, and the chance of intravascular mechanical thrombus extraction is even lower, which is closely related to the limited awareness of stroke of residents and primary medical staff in China, and the delay of pre-hospital treatment and hospital treatment $(7,8)$. Therefore, it is very important to improve the understanding of stroke among residents and medical staff. Our hospital is a national senior stroke center. Based on Zhengzhou Science and Technology Huimin Project, Zhengzhou residents and medical staff were given multi-level stroke education to improve their understanding of stroke and explore the impact on the treatment and prognosis of acute ischemic stroke.

\section{Patients and methods}

Study subjective. In this study, 519 patients with acute ischemic stroke were collected. They were treated in The 
First People's Hospital of Zhengzhou (Henan, China) from January 2018 to December 2018. Among them, 247 patients not from Guancheng district but in the neighborhood of The First People's Hospital of Zhengzhou were divided into the control group, including 132 males and 115 females aged $29-94$ years, with a mean age of $63.85 \pm 14.29$ and 272 cases from Guancheng district were divided into the experimental group, including 159 males and 113 females, aged 34-93 years, with a mean age of $64.03 \pm 14.51$.

Inclusion criteria: Patients in accordance with the diagnostic criteria in Guidelines for the diagnosis and treatment of acute ischemic stroke in China (3), and diagnosed with acute or ischemic stroke by CT or MR; Patients living in Zhengzhou for more than 2 years; for patients who were not from Guancheng district, they should have lived in the stroke treatment area of The First People's Hospital of Zhengzhou (1 h golden stroke time within the treatment circle), and their living area had high geographical homology with Guancheng district.

Exclusion criteria: patients with ischemic stroke who live beyond $1 \mathrm{~h}$ golden stroke treatment circle; stroke patients with DWI-negative or TIA; modified Rankin scale (MRS) before the onset grade $\geq 2$; patients with unknown onset time; patients with organ bleeding; patients with severe liver and kidney dysfunction; patients with disorder of consciousness and no relatives and patients who were unwilling to participate in the study.

The study was approved by the Ethics Committee of The First People's Hospital of Zhengzhou. Signed informed consents were obtained from the patients and/or guardians.

Education method. The treatment of stroke depends on the joint efforts of patients, their relatives, and medical staff to achieve good results. However, they play different roles in the treatment of stroke. Different methods of education should be used to improve efficiency. In January 2017, multi-level stroke education was started for residents and medical personnel in Guancheng district of Zhengzhou city. It lasted for about 1 year, and patients in non-Guancheng districts did not receive targeted publicity and education.

Medical staff education. Based on the Zhengzhou Guancheng district Health Committee, all medical staff within the jurisdiction were given education and training on stroke knowledge. Due to the different participation of different medical personnel in the treatment of stroke, they accepted different education programs, which were divided into three levels. Primary hospital doctors and community doctors play important roles in the treatment of stroke. They are involved in stroke education, stroke identification, stroke transfer, and secondary prevention of stroke. Primary stroke doctors and community doctors were trained in stroke definition, symptom recognition, stroke fast-cognition recognition, stroke first aid skills, and stroke secondary prevention. A total of eight sessions were held, each session was respectively evaluated. If the doctors failed the assessment, they entered the training again until they passed the assessment. Individual clinics and primary hospitals were trained in post stroke identification methods and stroke first aid flow charts, stroke emergency calls. The emergency center plays an important role in stroke identification and rapid transfer, and reached an agreement with the stroke emergency center to build a $1 \mathrm{~h}$ prime time treatment circle for stroke in The First People's Hospital of Zhengzhou. Stroke patients in the stroke treatment circle are preferentially transported to the hospital to reduce stroke rescue procedures, so the emergency doctors were mainly trained in the common symptoms of stroke, rapid recognition of stroke, first aid for stroke and stroke transport. The training was conducted every 3 months. Specialist doctors participated in the treatment of acute stroke in the whole process, and they were responsible for the education of patients, community doctors, primary doctors, and emergency doctors. Specialist doctors received more stringent trainings once a month. Specialists need to understand the frontier knowledge of stroke treatment, the latest stroke treatment guidelines. They should improve their professional skills and control the quality of stroke treatment cases, summarize the experience and lessons of stroke treatment, and continuously optimize the stroke treatment process.

Residents education. Residents' missions differ in the way they receive stroke knowledge due to differences in age and education. For example, young and middle-aged people are more likely to receive stroke education from the media and the internet. Older people may prefer newspapers, books, or even face-to-face talk. Therefore, a combination of multi-level education is used. Community doctors and primary hospital doctors enter the community for acute stroke education and distribute stroke treatment manuals (including stroke identification, stroke transport, stroke emergency call, secondary stroke prevention, prevention and control of stroke risk factors, healthy diet and exercise), once a week. Our hospital selects senior attending or title doctors to enter the community for stroke education and distribute stroke treatment manuals. Our hospital hold stroke seminars 5 times a week to promote stroke knowledge. Hospitalize stroke patients and their relatives can participate once or several times per week. Residents of Guancheng district are encouraged to participate. Media, we-media, television, internet and other channels are used to promote stroke knowledge.

Social platform. A social platform was developed for the treatment of stroke patients and stroke education, and the second line of thrombolysis ( $7 \times 24 \mathrm{~h}$ ) was responsible for consultation in the platform. The stroke treatment social platform mainly helps community doctors and emergency doctors in stroke identification, necessary examinations, and rapid transfer, and the handover between specialists and emergency medical services (EMS). The stroke education platform is mainly responsible for the residents' stroke consultation and education, guiding patients on exercise, diet, reasonable medication, and regular follow-up.

Questionnaire. A stroke questionnaire was designed by the second in line of stroke (senior professional title) according to the literature worldwide, to investigate the degree of stroke recognition. The contents included five aspects of stroke identification, stroke first aid method, patient transport route, awareness of intravenous thrombolysis, intravenous thrombolysis time window, awareness of intravascular mechanical thrombus. It was filled out by patients and immediate family members. 
Table I. General information of acute ischemic stroke.

\begin{tabular}{|c|c|c|c|}
\hline General information & Experimental group $(\mathrm{n}=272)$ & Control group $(\mathrm{n}=247)$ & P-value \\
\hline Age (years, mean $\pm \mathrm{SD}$ ) & $63.85 \pm 14.29$ & $64.03 \pm 14.51$ & 0.887 \\
\hline Systolic pressure $(\mathrm{mmHg}$, mean $\pm \mathrm{SD})$ & $163.99 \pm 107.05$ & $165.01 \pm 93.78$ & 0.998 \\
\hline $\mathrm{LDL}(\mathrm{mmol} / \mathrm{l}$, mean $\pm \mathrm{SD})$ & $2.98 \pm 1.08$ & $3.01 \pm 0.94$ & 0.449 \\
\hline NIHSS score & $7.31 \pm 5.84$ & $7.06 \pm 6.08$ & 0.634 \\
\hline Sex & & & 0.25 \\
\hline Male & 159 & 132 & \\
\hline Female & 113 & 115 & \\
\hline Hypertension & 165 & 155 & 0.625 \\
\hline Diabetes mellitus & 102 & 96 & 0.749 \\
\hline Coronary disease & 33 & 39 & 0.229 \\
\hline \multicolumn{4}{|l|}{ Personal history } \\
\hline Smoking & 132 & 109 & 0.316 \\
\hline Drinking & 72 & 67 & 0.866 \\
\hline Degree of education & & & 0.866 \\
\hline Primary school and below & 161 & 148 & \\
\hline Middle school and above & 111 & 99 & \\
\hline Ethnicity & & & 0.153 \\
\hline Hui & 31 & 19 & \\
\hline Han & 241 & 228 & \\
\hline
\end{tabular}

SD, standard deviation; LDL, low density lipoprotein NIHSS, National Institutes of Health Stroke Scale.

Observation index. The following indexes were recorded: Age, sex, educational level, understanding of stroke, the time from onset to hospital, the way of coming to hospital, the number of patients coming to hospital within $4.5 \mathrm{~h}$, the number of intravenous thrombus cases, door-to-needle time (DNT) and MRS score at 90 days, and the conversion cases of acute ischemic stroke hemorrhage. On the 90th day, MRS rating 0-2 suggested good prognosis, and MRS rating 3-6 suggested poor prognosis.

Statistical analysis. SPSS 17.0 (SPSS, Inc.) statistical software was used. If the measurement data were in accordance with normal distribution, t-test was used. If the measurement data was not in accordance with normal distribution, rank sum test was used. The counting data were tested by Chi-square test. $\mathrm{P}<0.05$ was considered statistically significant.

\section{Results}

General information on the subject of the survey. In this study, 519 patients with acute ischemic stroke were collected. The following data were recorded: Age, sex, hypertension, diabetes mellitus, NIHSS score at admission, personal history, ethnicity and educational level. There was no significant difference between the experimental and the control group by Chi-square test and independent sample t-test (Table I).

Stroke awareness. There was a difference in the discrimination degree of stroke symptoms between the experimental and the control group. There were significant differences in limb weakness (87.87 vs. 62.75\%), unclear speech (78.3 vs. 58.06\%), oblique mouth angle or facial paralysis (69.12 vs. 38.06\%), limb numbness ( 57.35 vs. $29.15 \%$ ), disturbance of consciousness (62.50 vs. $42.11 \%)$ and severe dizziness and walking instability (39.97 vs. $15.79 \%)$. The difference was statistically significant $(\mathrm{P}<0.05)$. There was no significant difference in visual acuity or binocular blindness, or severe headache $(\mathrm{P}>0.05)$.

There were statistically significant differences between the experimental and the control group in stroke treatment methods, such as EMS (35.29 vs. 17.81\%), seeing a doctor through other transportation (38.97 vs. 47.77\%), taking medicine by oneself or waiting at home (25.74 vs. $34.41 \%)$ $(\mathrm{P}<0.05)$. There were significant differences between the experimental and the control group in intravenous thrombolysis, intravenous thrombolysis time window and intravascular mechanical thrombus extraction, and there was significant difference between the experimental group and the control group $(\mathrm{P}<0.05)$ (Table II).

Treatment of acute ischemic stroke. In the experimental group, the shortest onset time was 20 min and the longest was $168 \mathrm{~h}$, with an average of $14.82 \pm 17.67 \mathrm{~h}$. In the control group, the shortest onset time was $36 \mathrm{~min}$ and the longest was $192 \mathrm{~h}$, with an average of $25.92 \pm 25.23 \mathrm{~h}$. The difference was statistically significant $(\mathrm{P}<0.05)$. Ninety-eight patients were transported by EMS in the experimental group, accounting for $36.02 \%$ of the patients with acute ischemic stroke, while 40 patients in the control group were transported by EMS, accounting for $16.19 \%$ 
Table II. Stroke awareness.

\begin{tabular}{|c|c|c|c|}
\hline Items & Experimental group $(n=272)$ & Control group $(n=247)$ & P-value \\
\hline \multicolumn{4}{|l|}{ Stroke identification } \\
\hline Limb weakness & $239(87.87 \%)$ & $155(62.75 \%)$ & $<0.001$ \\
\hline Unclear speech & $213(78.3 \%)$ & $136(55.06 \%)$ & $<0.001$ \\
\hline Oblique mouth angle or facial paralysis & $188(69.12 \%)$ & $94(38.06 \%)$ & $<0.001$ \\
\hline Limb numbness & $156(57.35 \%)$ & $72(29.15 \%)$ & $<0.001$ \\
\hline Unclear vision or binocular blindness & $37(13.6 \%)$ & $30(12.14 \%)$ & 0.621 \\
\hline Disturbance of consciousness & $170(62.5 \%)$ & $104(42.11 \%)$ & $<0.001$ \\
\hline Severe headache & $58(21.32 \%)$ & $49(19.83 \%)$ & 0.676 \\
\hline Severe dizziness and walking instability & $106(39.97 \%)$ & $39(15.79 \%)$ & $<0.001$ \\
\hline \multicolumn{4}{|l|}{ First aid methods for stroke } \\
\hline EMS & $96(35.29 \%)$ & $44(17.81 \%)$ & $<0.001$ \\
\hline Other ways of seeing a doctor & $176(64.71 \%)$ & $203(82.19 \%)$ & \\
\hline Understanding intravenous thrombolysis & $193(70.96 \%)$ & $58(23.48 \%)$ & $<0.001$ \\
\hline Understanding the time window of intravenous thrombolysis & $72(26.47 \%)$ & $16(6.48 \%)$ & $<0.001$ \\
\hline Understanding intravascular mechanical thrombus removal & $28(10.29 \%)$ & $11(4.45 \%)$ & 0.012 \\
\hline
\end{tabular}

EMS, emergency medical services.

Table III. Stroke treatment table.

\begin{tabular}{|c|c|c|c|}
\hline Assessment index & $\begin{array}{l}\text { Experimental group } \\
\qquad(\mathrm{n}=272)\end{array}$ & $\begin{array}{l}\text { Control group } \\
\quad(n=247)\end{array}$ & P-value \\
\hline Time from onset to arrival in hospital (h) & $14.82 \pm 17.67$ & $25.92 \pm 25.23$ & $<0.001$ \\
\hline Number of patients transferred by EMS & 98 & 40 & $<0.001$ \\
\hline Number of persons arriving in the hospital within $4.5 \mathrm{~h}$ & 67 & 32 & $<0.001$ \\
\hline Number of cases of intravenous thrombolysis & 55 & 17 & $<0.001$ \\
\hline Intravascular mechanical thrombus removal & 13 cases & 3 cases & $<0.001$ \\
\hline DNT time $(\mathrm{m})$ & $42.43 \pm 17.30$ & $63.35 \pm 26.53$ & $<0.001$ \\
\hline
\end{tabular}

DNT, door-to-needle time; EMS, emergency medical services.

of the patients with acute ischemic stroke, the difference was statistically significant $(\mathrm{P}<0.05)$.

Sixty-seven patients arrived in hospital within $4.5 \mathrm{~h}$ in the experimental group, accounting for $24.63 \%$ of the patients with acute ischemic stroke, while 32 patients in the control group had a gross onset of $4.5 \mathrm{~h}$, accounting for $12.96 \%$ of the patients with acute ischemic stroke, the difference was statistically significant.

The number of cases of venous thrombolysis in the experimental group was 55, accounting for $82.09 \%$ of the number of hospitalizations within $4.5 \mathrm{~h}$, accounting for $22.27 \%$ of the number of acute ischemic stroke. The number of cases of venous thrombolysis in the control group was 17 , accounting for $53.12 \%$ of the number of hospitalizations within $4.5 \mathrm{~h}$, accounting for $6.9 \%$ of the number of acute ischemic stroke. There was significant difference between the two groups $(\mathrm{P}<0.05)$.

There were 13 cases of intravascular mechanical thrombectomy in the test group, accounting for $4.78 \%$ of ischemic stroke, and 3 cases of intravascular mechanical thrombectomy in the control group, accounting for $1.21 \%$ of the number of acute ischemic stroke. The difference was statistically significant.

There was significant difference in DNT test between the experimental and the control group $(42.43 \pm 17.30$ vs. $63.35 \pm 26.53)$, and there was statistical significance $(\mathrm{P}<0.05)$ (Table III).

Recurrence and prognosis. In the experimental group, there were 128 patients with MRS0 grade and 102 patients with MRS1-2 grade (230 patients with good prognosis), accounting for $84.56 \%$ of patients with acute ischemic stroke. In the control group, there were 92 patients with MRSO grade and 77 patients with MRS1-2 grade (169 patients with good prognosis), accounting for $68.42 \%$ of patients with acute ischemic stroke. The difference between the two groups was statistically significant.

There were 11 cases of hemorrhagic transformation in the test group, accounting for $4.04 \%$ of acute ischemic stroke, 
Table IV. Prognosis

\begin{tabular}{lccc}
\hline $\begin{array}{l}\text { Prognosis } \\
\text { and outcome }\end{array}$ & $\begin{array}{c}\text { Experimental } \\
\text { group } \\
(\mathrm{n}=272)\end{array}$ & $\begin{array}{c}\text { Control } \\
\text { group } \\
(\mathrm{n}=247)\end{array}$ & P-value \\
\hline Favourable prognosis & 230 & 169 & $<0.001$ \\
Unfavourable prognosis & 42 & 78 & \\
Hemorrhagic transformation & 11 & 21 & 0.035 \\
Death & 12 & 18 & 0.161 \\
\hline
\end{tabular}

12 cases of death, accounting for $4.41 \%$ of ischemic stroke, 21 cases of hemorrhagic transformation in the control group, accounting for $8.50 \%$ of acute ischemic stroke, and 18 cases of death, accounting for $7.29 \%$ of ischemic stroke. There was a difference in hemorrhagic transformation after stroke between the two groups $(\mathrm{P}<0.05)$, and there was no difference in the number of deaths between the two groups $(\mathrm{P}>0.05)$ (Table IV).

\section{Discussion}

Acute ischemic stroke is the most common type of stroke, accounting for $70 \%$ of the cases. More than $60 \%$ of the patients still have different degrees of neurological dysfunction after treatment, only $1 / 3$ of the patients after treatment are completely asymptomatic (9-11). Restoring circulation for the purpose of intravenous thrombolysis or mechanical thrombolysis in acute treatment of ischemic stroke improved the prognosis of stroke patients, but the time window was narrow and the curative effect depends on time. In the United States, $<25 \%$ of eligible stroke patients arrive in the emergency department within $3 \mathrm{~h}$ of treatment, while in China, only $\sim 20 \%$ of the patients can reach the emergency department within $3 \mathrm{~h}$ of onset $(8,12)$. The effective measures to reduce the delay of stroke treatment include rapid stroke identification, rapid stroke transport, high efficiency treatment in hospital, and high cooperation between patients and their families $(13,14)$. With the construction of senior stroke center in China, hospital treatment and rapid transportation have been constantly improved, the rapid identification of stroke and the cooperation of patients still need continuous improvement. The identification of stroke by patients and relatives, and the adoption of rapid and reasonable transport measures were essential to enable patients to reach the hospital quickly. The rapid identification of stroke by primary medical staff and 120 medical staff enables the key factors to ensure the rapid transport of patients.

The recognition of early warning signs of stroke by the public is still low, and there are great differences in sex, age, race and living area (14-17). Studies worldwide have found that educational intervention can improve public recognition of stroke. The study of Morgenstern et al (18) found that propaganda and education on stroke among middle school students and their parents can significantly improve their stroke recognition ability and increase the thrombolysis rate of stroke patients. After a year of multi-level apoplexy education, it was found that the patients and their families in the experimental group had significantly improved the recognition ability of stroke, especially in the aspects of limb weakness, unclear speech, oblique or facial paralysis of mouth angle and disturbance of consciousness. Degree of recognition of limb weakness was the highest, and that of unclear speech was second. Patients and relatives have poor recognition of symptoms such as unclear vision and severe headache. There is no statistical significance compared with those after publicity and education. Considering the age of onset of the disease, it is related to the experience of eye diseases and headaches. The understanding of intravenous thrombolysis, intravascular mechanical thrombectomy and intravenous thrombolysis time window was significantly improved, the difference was statistically significant $(\mathrm{P}<0.05)$, but the understanding of thrombolysis time window and mechanical thrombectomy was still insufficient, and the proportion was low. More comprehensive and detailed stroke education may be needed to further improve residents' awareness of stroke.

Time is the brain. Emergency medical care service (EMS) is considered to be an effective way to quickly send patients to hospital. It could shorten the time for patients to arrive in hospital, and is an important means to improve the medical response and treatment of acute ischemic stroke (19-22). By EMS, hospitals can be notified in advance that patients who are likely to have stroke are being transported; stroke assessment team could act in advance and play an important role in increasing the number of patients undergoing thrombolysis, and can improve the prognosis of patients $(23,24)$. Patel et al (25) conducted a study of stroke patients in North Carolina $(n=13,894)$. It was found that compared with patients arriving by private transport, EMS pre-notice increased the number of patients receiving $\leq 25 \mathrm{~min}$ imaging and $\leq 45 \mathrm{~min}$ imaging reports, and more patients could receive thrombolysis. In this study, more patients in the experimental group used EMS (98 vs. 40) and more patients were treated with thrombolysis (55 vs. 17) than those in the control group, while $2 \%$ of the patients in the United States adopted emergency services. Compared with the United States, the utilization rate of emergency medical service is still low, so it is necessary to carry out various forms of stroke education, enhance the concept of using first aid service, so that patients with acute ischemic stroke can be treated in time and effectively in the shortest possible time, so as to reduce disability and improve the quality of life of patients. For strokes that have already occurred, patients should be transferred to a hospital capable of treatment as soon as possible, so that patients can be treated in a timely and reasonable manner. This is also the purpose of this article. Through multi-level stroke education, stroke symptoms can be identified timely, patients can be quickly transferred, green channels can be started in time, rapid diagnosis and medication can be used to enable patients to have a better prognosis.

Thrombolysis is the most effective treatment for acute ischemic stroke, but the time window is narrow and the thrombolysis rate is low. In an Australian study (26), only $14.7 \%$ of patients with acute ischemic stroke received thrombolysis within the time window ( $4.5 \mathrm{~h}$ after onset). From 2007 to 2008 , the proportion of thrombolysis in China's national stroke registration (27) was $1.9 \%$, accounting for $11.3 \%$ of the patients who arrived in emergency within $3 \mathrm{~h}$ after onset. In 2016, the National Health and Health Commission released a report showing that the proportion of patients receiving intravenous thrombolytic therapy was increased, accounting for $4.1 \%$ of all 
patients with ischemic stroke, but different regions, different levels of hospitals have a big difference (16). In this study, after education on stroke, the time from onset to hospital was reduced from $25.92 \pm 25.23 \mathrm{~h}$ in 2016 to $14.82 \pm 17.67 \mathrm{~h}$ on average, and the time to visit was greatly shortened. The number of patients arriving in hospital at $4.5 \mathrm{~h}$ accounted for $12.96 \%$ of all acute ischemic stroke, and increased to $24.63 \%$. The difference was statistically significant. In the United States, only $2.4-5.2 \%$ could arrive in hospital within $4.5 \mathrm{~h}$ among all patients with acute ischemic stroke. In the experimental group, 55 cases $(82.09 \%)$ received thrombolysis, accounting for $22.27 \%$ in acute ischemic stroke patients who arrived in hospital within $4.5 \mathrm{~h}$. While in the control group, 17 cases received thrombolysis, accounting for $53.12 \%$ among patients who arrived hospital within $4.5 \mathrm{~h}$, and accounting for $6.9 \%$ among all patients with acute ischemic stroke, which is better than those reported abroad, owing to our multi-level systematic education of stroke.

The sooner the blood vessels were opened and perfused, the prognosis of the patients was better. Since the construction of the stroke center in China, great achievements have been made in the treatment of stroke (28), from $125 \mathrm{~min}$ in 2013 to $54.8 \mathrm{~min}$ in 2017 , door-to-needle time (DNT) was shortened from $125 \mathrm{~min}$ in 2013 to $54.8 \mathrm{~min}$. In this study, the DNT of the experimental group and the control group $(42.43 \pm 17.30$ vs. $63.35 \pm 26.53)$ were significantly shortened, the prognosis of the experimental group was better ( 84.56 vs. $68.42 \%$ ), the bleeding conversion control group was decreased (4.04 vs. $8.50 \%$ ), and the mortality of the experimental group was not significantly different from that of the control group (4.41 vs. 7.29\%). It is suggested that multilevel stroke education can shorten the time of DNT, give the patients a better prognosis, and can reduce the transformation of bleeding after infarction, but cannot reduce mortality.

Collectively, multi-level systematic education can effectively improve the identification of stroke and the understanding of stroke treatment methods. It could promote the rapid transport of patients to hospital, so that more patients would be able to obtain intravenous thrombolysis or intravascular thrombus therapy, which may improve the prognosis of patients and reduce the transformation of bleeding after infarction. The sample of this study is small and the region is limited, so it still needs to be further studied. The First People's Hospital of Zhengzhou is located in the center of Zhengzhou city, at the junction of the three districts. Therefore, the residents in the neighborhood of The First People's Hospital of Zhengzhou (within the golden $1 \mathrm{~h}$ treatment time circle) were selected as the research subjects, which have a high degree of homology and excludes factors that interfere with the trial as much as possible. But the weather and road conditions at the time of onset, and residents' value on health may still have a certain impact on the treatment of stroke. Relevant data have not been collected in this study, and there are certain limitations in this study. Further research will be conducted. However, we believe that multi-level stroke education is beneficial to stroke treatment and worth promoting.

\section{Acknowledgements}

Not applicable.

\section{Funding}

No funding was received.

\section{Availability of data and materials}

The datasets used and/or analyzed during the present study are available from the corresponding author on reasonable request.

\section{Authors' contributions}

YL, XC and XZ conceived and designed the study. YL, XX, $\mathrm{YZ}, \mathrm{CW}$ and $\mathrm{XZ}$ were responsible for the collection and analysis of the experimental data. YL and $\mathrm{XC}$ interpreted the data and drafted the manuscript. YL and $\mathrm{XZ}$ revised the manuscript critically for important intellectual content. YL wrote the manuscript. All authors read and approved the final manuscript.

\section{Ethics approval and consent to participate}

The study was approved by the Ethics Committee of The First People's Hospital of Zhengzhou (Henan, China). Signed informed consents were obtained from the patients and/or guardians.

\section{Patient consent for publication}

Not applicable.

\section{Competing interests}

The authors declare that they have no competing interests.

\section{References}

1. Ojike N, Ravenell J, Seixas A, Masters-Israilov A, Rogers A, Jean-Louis G, Ogedegbe G and McFarlane SI: Racial disparity in stroke Awareness in the US: An analysis of the 2014 National Health Interview Survey. J Neurol Neurophysiol 7: 1-10, 2016.

2. Wang G, Fang B, Yu X and Li Z: Interpretation of 2018 guidelines for the early management of patients with acute ischemic stroke. Zhonghua Wei Zhong Bing Ji Jiu Yi Xue 30: 289-295, 2018 (In Chinese).

3. Zhao HM, Qin WQ, Wang PJ and Wen ZM: Eosinopenia is a predictive factor for the severity of acute ischemic stroke. Neural Regen Res 14: 1772-1779, 2019.

4. Furie KL and Jayaraman MV: 2018 guidelines for the early management of patients with acute ischemic stroke. Stroke 49: 509-510, 2018.

5. Nogueira RG, Jadhav AP, Haussen DC, Bonafe A, Budzik RF, Bhuva P, Yavagal DR, Ribo M, Cognard C, Hanel RA, et al; DAWN Trial Investigators: Thrombectomy 6 to 24 hours after stroke with a mismatch between deficit and infarct. N Engl J Med 378: 11-21, 2018.

6. Thomalla G, Simonsen CZ, Boutitie F, Andersen G, Berthezene Y, Cheng B, Cheripelli B, Cho TH, Fazekas F, Fiehler J, et al; WAKE-UP Investigators: MRI-Guided Thrombolysis for Stroke with Unknown Time of Onset. N Engl J Med 379: 611-622, 2018.

7. Zhao J, Zhao H, Li R, Li J, Liu C, Lv J, Li Y, Liu W, Ma D, Hao $\mathrm{H}$, et al: Outcome of multimodal MRI-guided intravenous thrombolysis in patients with stroke with unknown time of onset. Stroke Vasc Neurol 4: 3-7, 2019.

8. Nguyen-Huynh MN, Klingman JG, Avins AL, Rao VA, Eaton A, Bhopale S, Kim AC, Morehouse JW and Flint AC; KPNC stroke FORCE Team: Novel telestroke program improves thrombolysis for acute stroke across 21 hospitals of an integrated healthcare system. Stroke 49: 133-139, 2018. 
9. Mehta T, Strauss S, Beland D, Fortunato G, Staff I and Lee N: Stroke simulation improves acute stroke management: A systems-based practice experience. J Grad Med Educ 10: 57-62, 2018.

10. Fonarow GC, Zhao X, Smith EE, Saver JL, Reeves MJ, Bhatt DL, Xian Y, Hernandez AF, Peterson ED and Schwamm LH: Doorto-needle times for tissue plasminogen activator administration and clinical outcomes in acute ischemic stroke before and after a quality improvement initiative. JAMA 311: 1632-1640, 2014.

11. Ruff IM, Liberman AL, Caprio FZ, Maas MB, Mendelson SJ Sorond FA, Bergman D, Bernstein RA, Curran Y and Prabhakaran S: A resident boot camp for reducing door-toneedle times at academic medical centers. Neurol Clin Pract 7 : 237-245, 2017.

12. Ekundayo OJ, Saver JL, Fonarow GC, Schwamm LH, Xian Y, Zhao X, Hernandez AF, Peterson ED and Cheng EM: Patterns of emergency medical services use and its association with timely stroke treatment: Findings from Get With the Guidelines-Stroke. Circ Cardiovasc Qual Outcomes 6: 262-269, 2013.

13. Rostanski SK, Stillman JI, Schaff LR, Perdomo CA, Liberman AL, Miller EC, Marshall RS, Willey JZ and Williams O: E-mail is an effective tool for rapid feedback in acute stroke. Neurohospitalist 7: 159-163, 2017.

14. Sharobeam A, Jones B, Walton-Sonda D and Lueck CJ: Factors delaying intravenous thrombolytic therapy in acute ischaemic stroke: A systematic review of the literature. J Neurol: Mar 21, 2020 (Epub ahead of print). doi: 10.1007/s00415-020-09803-6.

15. Dong Y and Dong Q: Current status of intravenous thrombolysis in acute ischemic stroke in China. Zhongguo Shiyong Neike Zazhi 38: 685-688, 2018 (In Chinese).

16. Mochari-Greenberger H, Xian Y, Hellkamp AS, Schulte PJ, Bhatt DL, Fonarow GC, Saver JL, Reeves MJ, Schwamm LH and Smith EE: Racial/ethnic and sex differences in emergency medical services transport among hospitalized US stroke patients: Analysis of the national get with the guidelines-stroke registry. J Am Heart Assoc 4: e002099, 2015.

17. Fatovich DM: A population-based study of thrombolysis for acute stroke in South Australia. Med J Aust 194: 431-432, 2011.

18. Morgenstern LB, Bartholomew LK, Grotta JC, Staub L, King M and Chan W: Sustained benefit of a community and professional intervention to increase acute stroke therapy. Arch Intern Med 163: 2198-2202, 2003

19. Lin CB, Peterson ED, Smith EE, Saver JL, Liang L, Xian Y, Olson DM, Shah BR, Hernandez AF, Schwamm LH, et al: Emergency medical service hospital prenotification is associated with improved evaluation and treatment of acute ischemic stroke. Circ Cardiovasc Qual Outcomes 5: 514-522, 2012.
20. Berglund A, Svensson L, Sjöstrand C, von Arbin M, von Euler M, Wahlgren N, Engerström L, Höjeberg B, Käll TB, Mjörnheim S, et al; HASTA Collaborators: Higher prehospital priority level of stroke improves thrombolysis frequency and time to stroke unit: The Hyper Acute STroke Alarm (HASTA) study. Stroke 43: 2666-2670, 2012.

21. Jin H, Zhu S, Wei JW, Wang J, Liu M, Wu Y, Wong LK, Cheng Y, Xu E, Yang Q, et al; ChinaQUEST (Quality Evaluation of Stroke Care and Treatment) Investigators: Factors associated with prehospital delays in the presentation of acute stroke in urban China. Stroke 43: 362-370, 2012.

22. Wireklint Sundström B, Andersson Hagiwara M, Brink P, Herlitz J and Hansson PO: The early chain of care and risk of death in acute stroke in relation to the priority given at the dispatch centre: A multicentre observational study. Eur J Cardiovasc Nurs 16: 623-631, 2017.

23. Abdullah AR, Smith EE, Biddinger PD, Kalenderian D and Schwamm LH: Advance hospital notification by EMS in acute stroke is associated with shorter door-to-computed tomography time and increased likelihood of administration of tissueplasminogen activator. Prehosp Emerg Care 12: 426-431, 2008.

24. Zerwic J, Hwang SY and Tucco L: Interpretation of symptoms and delay in seeking treatment by patients who have had a stroke: Exploratory study. Heart Lung 36: 25-34, 2007.

25. Patel MD, Rose KM, O'Brien EC and Rosamond WD: Prehospital notification by emergency medical services reduces delays in stroke evaluation: Findings from the North Carolina stroke care collaborative. Stroke 42: 2263-2268, 2011.

26. Eissa A, Krass I, Levi C, Sturm J, Ibrahim R and Bajorek B: Understanding the reasons behind the low utilisation of thrombolysis in stroke. Australas Med J 6: 152-167, 2013.

27. Wang Y, Liao X, Zhao X, Wang DZ, Wang C, Nguyen-Huynh MN, Zhou Y, Liu L, Wang X, Liu G, et al: China National Stroke Registry Investigators: Using recombinant tissue plasminogen activator to treat acute ischemic stroke in China: Analysis of the results from the Chinese National Stroke Registry (CNSR). Stroke 42: 1658-1664, 2011.

28. Wang L: Current status and future of stroke prevention and treatment projects in China. J Second Mil Med Univ 39: 941-943, 2018. 